\title{
Comparative Analysis of Dispersion Compensating Fiber (DCF) and Optical Phase Conjugation (OPC) used for Dispersion Compensation
}

\author{
Aradhana Asiwal ${ }^{1}$, Divya Dhawan ${ }^{2}$ \\ Research Scholar, Electronics and Communication Engineering, PEC university of technology, Chandigarh, India ${ }^{1}$ \\ Assistant Professor, Electronics and Communication Engineering, PEC university of technology, Chandigarh, India ${ }^{2}$
}

\begin{abstract}
Various techniques are used for the dispersion compensation of the fiber and two of them are Dispersion Compensating Fiber (DCF) and Optical Phase Conjugation (OPC). In this paper the performance of the system is analyzed by comparing various results at the receiver. A 10Gb/s NRZ signal is launched onto a $100 \mathrm{~km}$ long Single Mode Fiber (SMF). Then fiber length is increased by increasing number of fiber spans. The length is varied up to 500 $\mathrm{km}$. Results in the terms of eye diagram, BER, Q-factor are shown and it can be seen that results of a system which consist of OPC are enhanced because of proper dispersion compensation.
\end{abstract}

Keywords: Dispersion Compensating Fiber (DCF), Optical Phase Conjugation (OPC), Single Mode Fiber (SMF).

\section{INTRODUCTION}

Due to demand of internet in daily life, there is a rapid growth in communication technologies like optical communication which have some promising advantages in terms of bandwidth, input power and losses in the channel over other communication forms. But in optical communication, dispersion and nonlinearities are two major problems faced by systems installed worldwide. The reliability of optical system is affected by broadening of pulse called dispersion which causes Inter-Symbol Interference (ISI) and it limits the length and capacity of the transmission system.

Optical system performance degrades by chromatic dispersion (CD) with length of fiber more than $100 \mathrm{kms}$. To compensate dispersion few techniques have been proposed for optical system[1], and one them is Optical phase conjugation which uses phase conjugated wave to compensate dispersion at the receiver and is innovative over other compensation techniques like DCF and Fiber Bragg Grating (FBG), etc.

\section{DISPERSION COMPENSATION TECHNIQUES}

A. Dispersion Compensating Fiber (DCF)

DCF is known to be a good technology for the compensation of dispersion. The positive dispersion of the conventional fiber is reduced by large negative dispersion coefficient of DCF. Proper length of DCF is required which can compensate the dispersion of conventional fiber.

Depending on the position of the DCF, Compensation is done by three different methods:

A) Pre-Compensation

B) Post-Compensation

C) Symmetrical-Compensation

A DCF must have small insertion loss, low optical nonlinearity, small polarization mode dispersion and it should also have large chromatic dispersion coefficient to decrease the size of DCF. Always a small size of the DCF is better. Net dispersion is reduced to zero if a DCF with negative dispersion is placed after a SMF with positive dispersion.

$$
\mathrm{D}_{\mathrm{SMF}} \times \mathrm{L}_{\mathrm{SMF}}=-\mathrm{D}_{\mathrm{DCF}} \times \mathrm{L}_{\mathrm{DCF}}
$$

Where $\mathrm{D}$ is the dispersion and $\mathrm{L}$ is length of each fiber respectively [2]. 
UGC Approved Journal

IJIREEICE

Vol. 5, Issue 7, July 2017

B. Optical Phase Conjugation (OPC)

Optical phase conjugation (OPC), also referred as Mid Span spectral inversion (MSSI), is a promising technology firstly proposed by Yariv et al. in 1979 to mitigate impairments in long-haul communication systems such as Kerr effect and chromatic dispersion [3][4]. OPC is placed at the mid of the fiber link so that the distorted signal after passing through the first fiber span get compensated after passing through OPC and subsequent propagation through second fiber span [5].

The principle of operation for OPC block is phase inversion according to:

$$
\begin{aligned}
& \operatorname{Ein}(t)=A(t) e^{j \Phi(t)} \\
& \operatorname{Eout}(t)=\eta A(t) e^{-j \Phi(t)} e^{j \Phi s h i f t}
\end{aligned}
$$

Where $\eta$ is device efficiency, фshift is extra phase shift. Here $\phi$ shift is equal to $\pi / 2$ and $\eta=1$ [6][7].

In this paper, the use of DCF and OPC is proposed to compensate the CD in $100 \mathrm{~km}$ long Single Mode Fiber (SMF). The fiber length is increased to $500 \mathrm{~km}$ by increasing the number of fiber spans. Simulation results show that, when OPC is compared with DCF, improvement in Q-factor achieved is at least 3.47.

\section{SIMULATION SETUP}

Fig. 1, Fig. 2, Fig 3 and Fig 4 show the simulation setup of optical communication system. Simulation is carried out using Optsim software to demonstrate the effect of various dispersion compensation techniques and the results are being compared.

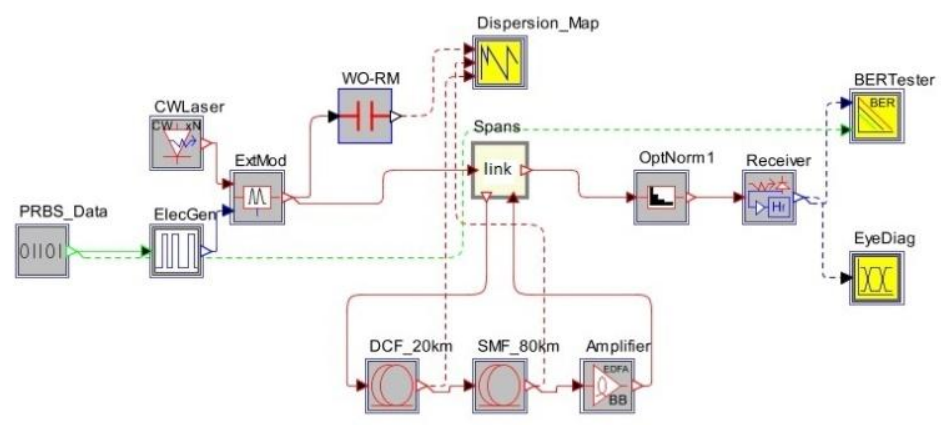

Fig. 1 Simulation setup of an optical communication system consisting of Pre-DCF

Simulation setup of an optical communication system is designed in which transmitter consists of a PRBS (Pseudo Random Bit Sequence) generator which transmits data at a bit rate of 10Gbps. PRBS transmit the data to Electrical Signal Generator which uses a NRZ modulation and a voltage signal. A continuous wave $(\mathrm{CW})$ laser with external modulation at a wavelength of $1550 \mathrm{~nm}$ is used as a source. Laser power used is $0 \mathrm{dBm}(1 \mathrm{~mW})$. Data from Electrical Signal Generator and continuous wave laser is transmitted to Electro absorption modulator where it gets modulated using Mach Zehnder modulator. The modulated signal is transmitted through a transmission distance of $100 \mathrm{~km}$. Transmission distance consist of single mode fiber (SMF) whose length is $80 \mathrm{~km}$ and Dispersion compensation fiber (DCF) whose length is $20 \mathrm{~km}$. Then this transmission length is increased by increasing the fiber spans. Transmission distance is increased to $500 \mathrm{~km}$.

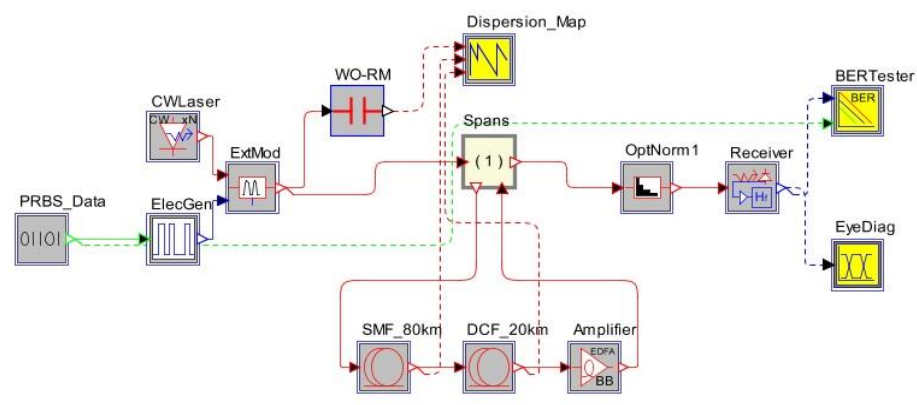

Fig. 2 Simulation setup of an optical communication system consisting of Post-DCF 
UGC Approved Journal

IJIREEICE

Vol. 5, Issue 7, July 2017

Loss in single mode fiber is $0.25 \mathrm{~dB} / \mathrm{km}$ and dispersion slope is $90 \mathrm{~S} / \mathrm{m}^{3}$ and loss in Dispersion compensating fiber is $0.5 \mathrm{~dB} / \mathrm{Km}$ and dispersion slope is $-360 \mathrm{~S} / \mathrm{m}^{3}$. The positive dispersion of SMF is compensated by negative dispersion of DCF. Nonlinearity factor and diameter of SMF and DCF are $2.6 \mathrm{e}^{-20} \mathrm{~m}^{2} / \mathrm{W}$ and $8.2 \mathrm{e}^{-6} \mathrm{~m}$ respectively. Data is transmitted through the fiber and received at the receiver. Before receiver the data is passed through the optical power normalizer whose average output power is $-22 \mathrm{dBm}$. Receiver with low pass filter and $1 \mathrm{GHz}$ bandwidth is used followed by a BER tester. Eye diagram analyzer is also used. Optical Phase Conjugation (OPC) is also used in place of DCF for dispersion compensation. It is used in the middle of the fiber link as shown in Fig. 4. The length of fiber before and after OPC is same to mitigate the dispersion more effectively. Efficiency of the OPC is chosen as 1 to acquire best results.

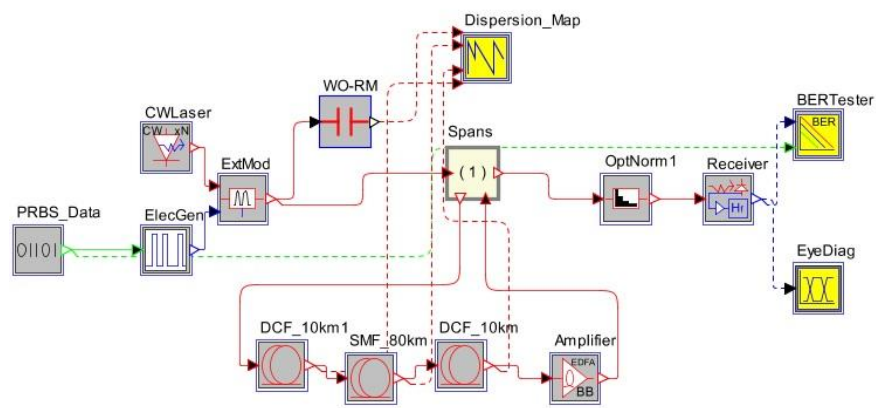

Fig. 3 Simulation setup of an optical communication system consisting of Symmetrical-DCF

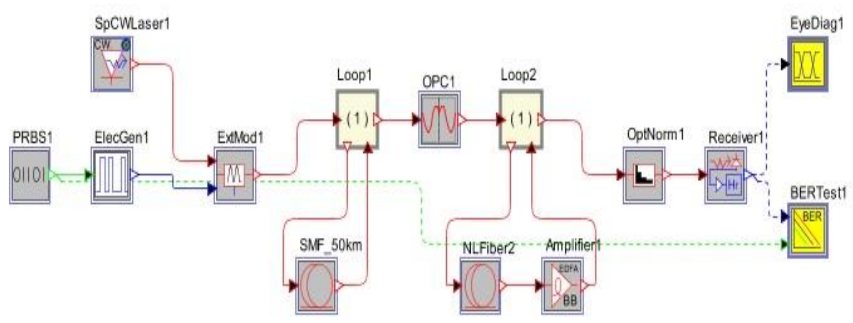

Fig. 4 Simulation setup of an optical communication system consisting of OPC

\section{SIMULATION RESULTS}

Comparison of various techniques in terms of eye diagram, Q-factor, and BER is done. Fig.5 shows the eye opening of Pre-DCF, Post-DCF, Symmetrical DCF and OPC. Eye opening of Pre-, Post- and Symmetrical-DCF is almost same. Maximum Q-factor and minimum BER is achieved for OPC and it is proved to be best technique because residual dispersion and high order derivatives are completely removed using this technique.

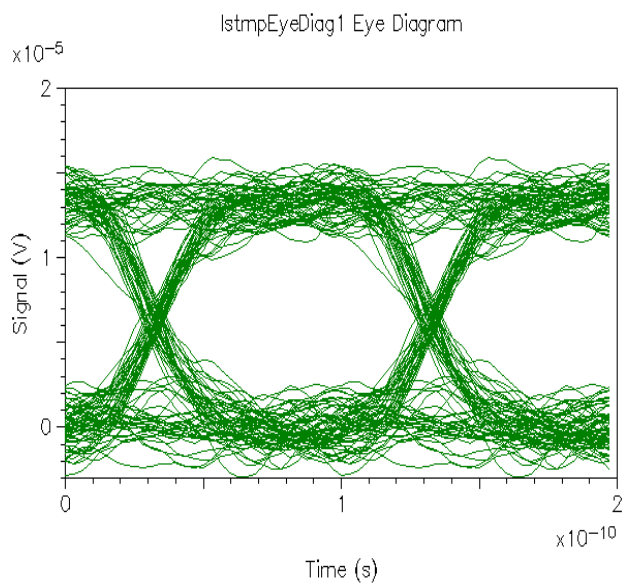

(a) 
UGC Approved Journal

IJIREEICE

International Journal of Innovative Research in

Electrical, Electronics, Instrumentation and Control Engineering

ISO 3297:2007 Certified

Vol. 5, Issue 7, July 2017

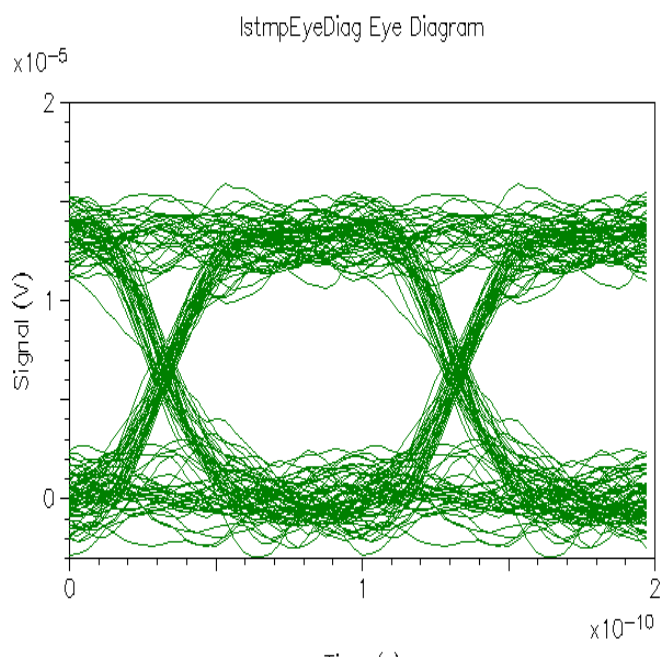

Time (s)

(b)

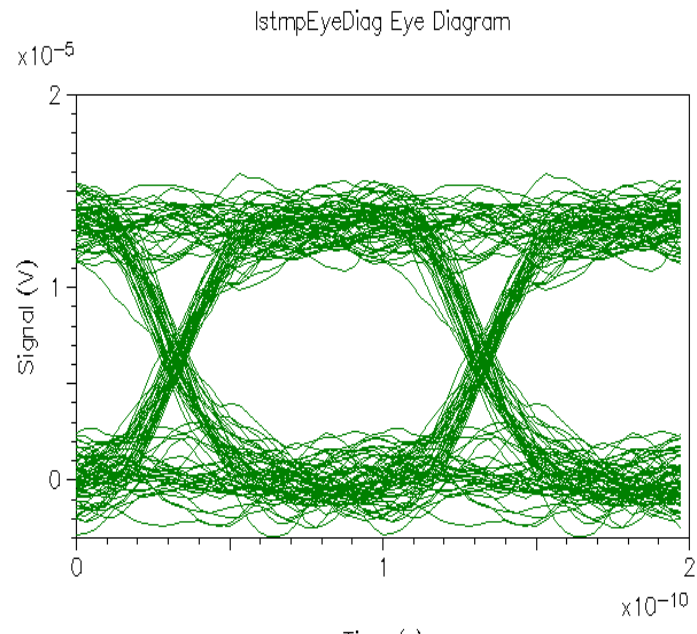

Time (s)

(c)

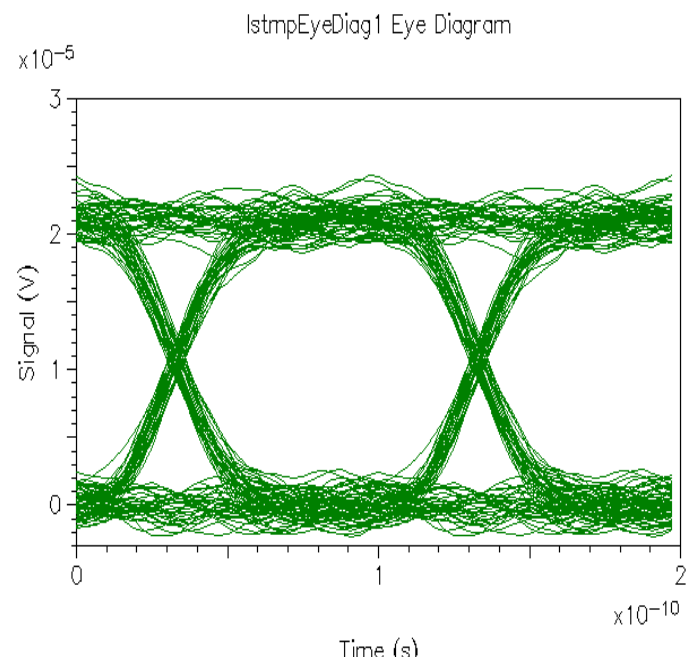

(d)

Fig. 5 Eye Opening of (a) Pre-DCF, (b) Post-DCF, (c) Symmetrical-DCF and (d) OPC 
UGC Approved Journal

IJIREEICE

Vol. 5, Issue 7, July 2017

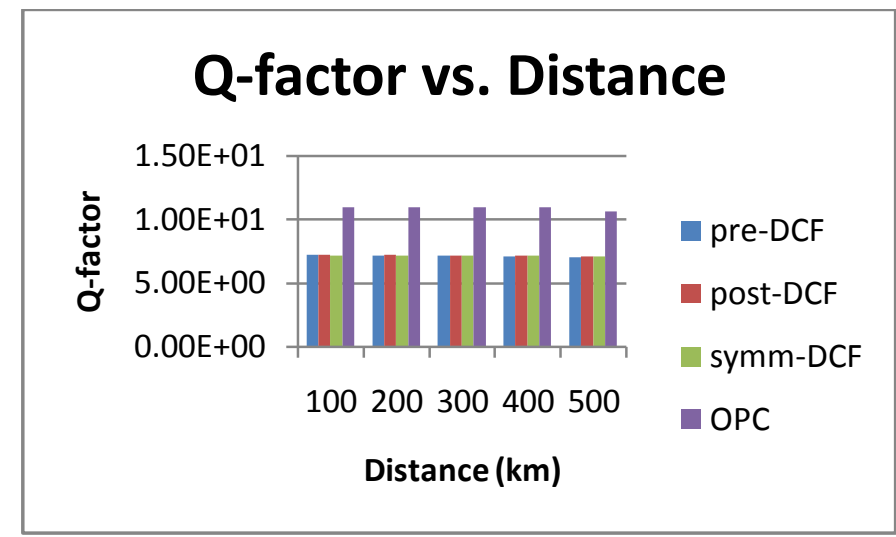

Fig. 6 Q-factor of different dispersion compensation techniques

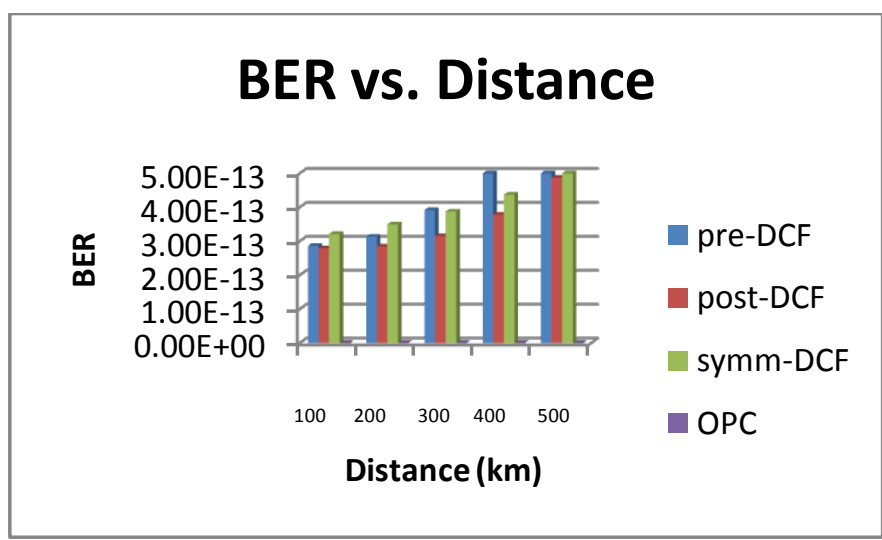

Fig. 7 BER of different dispersion compensation techniques

\section{RESULTS AND DISCUSSION}

The performance of the system is analyzed in terms of Eye-Opening, Q-factor and BER.

Q-Factor - It tells about the qualitative behavior of a digital signal in any system. It is denoted by $\mathrm{Q}$ and it is a dimensionless quantity. It is expressed as Signal-to-Noise Ratio[8].

\section{Where}

$$
\text { OSNR }=10 \log \left[\frac{\mathrm{P}_{\mathrm{i}}}{\mathrm{N}_{\mathrm{i}}}\right]+10 \log \left[\frac{\mathrm{B}_{\mathrm{m}}}{\mathrm{B}_{\mathrm{r}}}\right]
$$

$\mathrm{P}_{\mathrm{i}}$ - average power of the optical signal $\mathrm{i}_{\text {th }}$ channel $[\mathrm{W}]$

$\mathrm{N}_{\mathrm{i}}$ - interpolated value of the average noise power [W]

$\mathrm{B}_{\mathrm{m}}$ - Spectral width, in which we measure [nm]

$\mathrm{B}_{\mathrm{r}}$ - Reference Bandwidth

Bit Error Rate - It is the number of bit errors divided by total number of transferred bits during a time interval. It is expressed as

$$
\mathrm{BER}=\frac{\mathrm{n}_{\mathrm{c}}}{\mathrm{n}_{\mathrm{B}}}
$$

Where

$\mathrm{n}_{\mathrm{c}}=$ number of bits received

$\mathrm{n}_{\mathrm{B}}=$ total number of bits received in the defined time interval

BER can also be expressed in terms of Q-Factor as

$$
\mathrm{BER}=\frac{1}{2}\left[\frac{1}{\sqrt{2 \pi}} * \frac{1}{\mathrm{Q}} * \mathrm{e}^{-\frac{\mathrm{Q}^{2}}{2}}\right]
$$

Eye Opening - Eye diagram of an optical system displays the digital signal at the receiver. The width of the eye opening gives a measure of time interval over which the received signal can be sampled without error due to interference from adjacent pulses. The height of the eye opening gives an idea about the noise level. 


\section{International Journal of Innovative Research in Electrical, Electronics, Instrumentation and Control Engineering}

\section{ISO 3297:2007 Certified}

Vol. 5, Issue 7, July 2017

Fig. 5 shows eye opening of various techniques at $0 \mathrm{dBm}(1 \mathrm{~mW})$ laser power and $100 \mathrm{~km}$ fiber length. Eye of OPC is much wider as compared to eye of Pre-DCF, Post-DCF and Symmetrical-DCF because it is placed in the middle of fiber link. Using OPC, dispersion is compensated by reversing the propagation direction and phase variation of beam light. There is very little variation in eye opening of Pre-DCF, Post-DCF and Symmetrical-DCF. Therefore, results of OPC are better than DCF in terms of eye opening. As the transmission distance increases, the opening of eye decreases.

Fig. 6 shows the Q-factor of Pre-, Post-, Symmetrical-Compensation and OPC. Q-factor of Pre-, Post-, Symmetricalcompensation is 7.21, 7.21 and 7.19 respectively at $100 \mathrm{~km}$ transmission distance and 7.05, 7.13 and 7.13 respectively for $500 \mathrm{~km}$ transmission distance which are nearly same but for OPC, Q-factor is 10.96 and 10.60 for $100 \mathrm{~km}$ and 500 $\mathrm{km}$ respectively which is approximately 3.77 and 3.47 more than that of DCF for $100 \mathrm{~km}$ and $500 \mathrm{~km}$ transmission distance. The Q-Factor of OPC is better than that of DCF because OPC is immune to residual dispersion and as it is implemented in the middle of the transmission link, the effect of dispersion is equalized [9].

The results of DCF and OPC in terms of BER are shown in Fig. 7. BER of Pre-, Post-, Symmetrical-compensation is $2.86 \mathrm{e}^{-13}, 2.79 \mathrm{e}^{-13}$ and $3.21 \mathrm{e}^{-13}$ respectively for $100 \mathrm{~km}$ transmission distance and $8.59 \mathrm{e}^{-13}, 4.87 \mathrm{e}^{-13}$ and $5.01 \mathrm{e}^{-13}$ respectively for $500 \mathrm{~km}$ transmission distance whereas BER of OPC is $2.99 \mathrm{e}^{-28}$ and $1.46 \mathrm{e}^{-26}$ for $100 \mathrm{~km}$ and $500 \mathrm{~km}$ respectively. This clearly shows that BER achieved for OPC is very much less than that of DCF. Therefore, OPC is found to be the best technique among others as it has minimum BER and maximum Q-factor [10].

\section{CONCLUSION}

Though DCF is a good technique for dispersion compensation but because of certain disadvantages like high insertion loss, bulkiness, limited input power, presence of residual dispersion, high order derivatives, high attenuation, low negative dispersion, etc., OPC is more suitable and reliable for the compensation. When OPC is used results in terms of Q-factor, BER and eye opening are improved to a greater extent. Many studies are accepted in this perspective to reduce the effect of dispersion to large extent by introducing new system design.

\section{REFERENCES}

[1] E. Torrengo et al., "Influence of Pulse Shape in 112-Gb / s WDM PDM-QPSK Transmission," vol. 22, no. 23, pp. 1714-1716, 2010.

[2] D. Dhawan and N. Gupta, "OPTIMIZATION OF FIBER BASED DISPERSION COMPENSATION IN RZ AND NRZ DATA MODULATION FORMATS Abbreviations 2 . Dispersion Compensation Schemes Employed,” vol. 6, no. 6, pp. 651-663, 2011.

[3] A. Yariv, D. Fekete, and D. M. Pepper, "Compensation for channel dispersion by nonlinear optical phase conjugation," vol. 4, no. 2, pp. 52-54, 1979.

[4] L. Li, Y. Qiao, and Y. Ji, “Optimized optical phase conjugation conf iguration for f iber nonlinearity compensation in CO-OFDM systems,” vol. 9, no. 6, pp. 6-10, 2011

[5] X. Liu, Y. Qiao, and Y. Ji, "Reduction of the fi ber nonlinearity impairment using optical phase conjugation in 40 Gb / s CO-OFDM systems," OPTICS, vol. 283, no. 13, pp. 2749-2753, 2010.

[6] K. Bhowmik, M. Ahamed, and A. Momin, "Reduction of Dispersion in Optical Fiber Communication by Fiber Bragg Grating and Optical Phase Conjugation Techniques," vol. 2, no. 3, pp. 49-58, 2012

[7] A. Asiwal and D. Dhawan, "Dispersion Compensation and Nonlinearity Mitigation Using Optical Phase Conjugation and Other Techniques- A Review," vol. 5, no. 10, 2016.

[8] T. Ivaniga and P. Ivaniga, "Evaluation of the bit error rate and Q-factor in optical networks," vol. 9, no. 6, pp. 1-3, 2014.

[9] S. S. Dabhade and S. Bhosale, "Fiber Bragg Grating And Phase Conjugator As Dispersion Compensator,” no. 1, pp. 15-19, 2012.

[10] P. Shukla and K. P. Kaur, "Comparison of Pre- , Post- , and Symmetrical Dispersion Compensation Schemes for 40 Gbps NRZ data at Single \& Multiple Channels,” vol. 7109, pp. 101-105, 2013.

\section{BIOGRAPHIES}

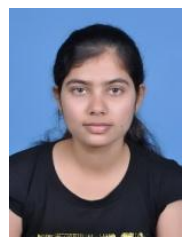

Aradhana Asiwal pursuing M.Tech. in the branch of Electronics Engineering at PEC University of Technology Chandigarh, India.

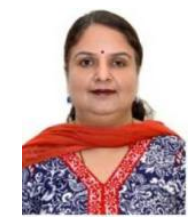

Mrs. Divya Dhawan is currently working as Assistant Professor in ECE Department of PEC University of Technology Chandigarh, India. She has a teaching experience of nearly 18 years. She is a member of IEEE, ISTE, and IEI. Her research interests include Optical Communication, Photonics Systems and Digital system design. 